\title{
An improved approximation for the analytical treatment of the local linear gyro-kinetic plasma dispersion relation in toroidal geometry
}

\author{
P. Migliano*1, D. Zarzoso ${ }^{1}$, F.J. Artola ${ }^{1}$, Y. Camenen ${ }^{1}$ and X. Garbet ${ }^{2}$ \\ ${ }^{1}$ Aix-Marseille Université, CNRS, PIIM UMR 7345, 13397 Marseille, France \\ ${ }^{2}$ CEA, IRFM, F-13108 St. Paul-lez-Durance cedex, France
}

\begin{abstract}
The analytical treatment of plasma kinetic linear instabilities in toroidal geometry is commonly tackled employing a power series expansion of the resonant part of the dispersion relation. This expansion is valid under the assumption that the modulus of the mode frequency is smaller than the magnitude of the frequencies characterizing the system (the drift, bounce and transit frequencies for example). We will refer to this approximation as High Frequency Approximation (HFA). In this paper the linear plasma dispersion relation is derived in the framework of the gyro-kinetic model, for the electrostatic case, in the local limit, in the absence of collisions, for a non rotating plasma, considering adiabatic electrons, in toroidal circular geometry, neglecting the parallel dynamics effect. A systematic analysis of the meaning and limitations of the HFA is performed. As already known, the HFA is not valid for tokamak relevant parameters. A new way to approximate the resonant part of the dispersion relation, called here Improved High Frequency Approximation (IHFA), is therefore proposed. A quantitative analysis of the Ion Temperature Gradient (ITG) instability is presented. The IHFA is shown to be applicable to the treatment of the ITG instability in tokamaks.
\end{abstract}

The solution of the dispersion relation for electrostatic field modes is one of the oldest problem in plasma physics. It is of fundamental importance for the prediction of transport levels in magnetically confined plasmas. Nowadays, this calculation can be performed numerically in many different physical scenarios and, thanks to the computational power available, the estimation of the linear mode frequency and growth rate is fast and accurate. However, the analytical treatment of the system remains appealing, since it provides a way to understand the underlying physics, investigate new possible physical mechanisms and verify the consistency of the numerical results.

The High Frequency Approximation (HFA), see e.g. $[1,2]$, provides a way for a fully analytical approach to the problem. It relies on the assumption that the modulus of the mode frequency considered is smaller than the magnitude of the frequencies characterizing the system (the drift, bounce and transit frequencies for example) allowing a power series expansion of the resonant part of the disper-

*pierluigi.migliano@univ-amu.fr sion relation. This approximation has been and still is widely employed for the description of linear modes instabilities in plasma physics, see e.g. $[3,4,5,6,7,8,9]$. It is in fact a very useful and powerful tool to understand the qualitative behaviour of the system under study, given its simplicity of use. However, it is a very constraining approximation and a lot of care has to be taken in its application when choosing the range of parameters.

In this paper the linear plasma dispersion relation is derived in the framework of the gyro-kinetic model [10, 11, 12, 13], for the electrostatic case, in the local limit [14], in the absence of collisions, for a non rotating plasma, considering adiabatic electrons, in toroidal circular geometry [15], neglecting the parallel dynamics effect. A systematic analysis of the meaning and limitations of the HFA applied to the Ion Temperature Gradient (ITG) instability is performed. This analysis elucidates the reason why the HFA is not valid for tokamak relevant scenarios (as stated in [1, 2]). A new way to approximate the resonant part of the dispersion relation is then proposed, it will be called Improved High Frequency Approximation (IHFA). It allows an ana- 
lytical treatment of the dispersion relation through a power series expansion which is applicable under less restrictive conditions compared to the HFA. It is shown that the IHFA can be used to study tokamak relevant range of parameters.

One interest of having an analytical expression for the ITG modes dispersion relation is to be able to build models describing their coupling to different modes. For example, it has been shown in [16] through numerical simulations that ITG modes and EGAM can interact with each other. In [17, 18] an analytical expression for the EGAM dispersion relation is given. An analytical treatment of the ITG modes applicable to experimentally relevant scenarios would then allow the modelling and investigation of the mechanism generating this interaction [19]. Furthermore, we stress the fact that the analysis presented in this paper is applicable to the general treatment of linear plasma instabilities since it relies only on the mathematical form of the resonant part of the dispersion relation. Therefore, although only the ITG instability is quantitatively studied, all the comments about the difficulties and limitations of the HFA and the possibility of a proper analytical treatment given by the IHFA are of general validity.

The linear dispersion relation is derived in what follows. Gyro-center toroidal coordinates $\left(\mathbf{X}, v_{\|}, \mu\right)$ are used, $\mathbf{X}=(r, \theta, \varphi)$ being the gyro-center position with $r, \theta$ and $\varphi$ respectively radial, poloidal and toroidal coordinates. The velocity space coordinates are the gyro-center velocity component parallel to the magnetic field $v_{\|}$and the magnetic moment $\mu=m v_{\perp}^{2} /(2 B)$, with $m$ the ion mass, $v_{\perp}$ the gyro-center velocity component perpendicular to the magnetic field and $B$ the magnetic field strength evaluated at the low field side position of the flux surface considered. The calculation is performed in circular geometry [15]. The circular geometry equilibrium assumes circular and concentric flux surfaces with the poloidal flux being a function of the radial coordinate only. In the electrostatic case, this is a valid assumption when considering only first order $\epsilon$ (the inverse aspect ratio) corrections to the geometry terms. Despite this fact, the various geometry terms are not expanded in $\epsilon$ in the following calculations. This choice is motivated both by simplicity and by the fact that this is the same procedure applied in the gyro-kinetic code GKW [20], which is used in this paper for comparison with the results of the analytical calculations. However, one has to remember that the results are only valid up to first order in $\epsilon$ (i.e. $\epsilon \ll 1$ ). The equilibrium magnetic field for circular geometry is given by

$$
\mathbf{B}=\frac{B_{0} R_{0}}{R}\left(\mathbf{e}_{\varphi}+\mathbf{e}_{\theta} \frac{\epsilon}{\bar{q}}\right)
$$

where $B_{0}$ is the magnetic field strength evaluated at the magnetic axis, $R=R_{0}+r \cos \theta$ with $R_{0}$ the tokamak major radius, $\mathbf{e}_{\varphi}$ and $\mathbf{e}_{\theta}$ are the unit vectors in the toroidal and poloidal direction respectively, $\bar{q}$ is a parameter related to the safety factor $q$ by

$$
q=\frac{1}{2 \pi} \int_{0}^{2 \pi} \frac{\mathbf{B} \cdot \mathbf{e}_{\varphi}}{\mathbf{B} \cdot \mathbf{e}_{\theta}} d \theta=\frac{\bar{q}}{\sqrt{1-\epsilon^{2}}}
$$

The parallel and perpendicular gradient operators are given respectively by

$$
\begin{aligned}
\nabla_{\|}= & \mathbf{b}(\mathbf{b} \cdot \nabla)=\mathbf{b} \frac{1}{\mathcal{F}}\left(\frac{1}{R} \partial_{\varphi}+\frac{1}{\bar{q} R_{0}} \partial_{\theta}\right) \\
\nabla_{\perp}= & \nabla-\nabla_{\|}=\mathbf{e}_{r} \partial_{r}+ \\
& +\mathbf{e}_{\theta}\left[\frac{1}{r} \partial_{\theta}-\frac{\epsilon}{\bar{q} \mathcal{F}^{2}}\left(\frac{1}{R} \partial_{\varphi}+\frac{1}{\bar{q} R_{0}} \partial_{\theta}\right)\right]+ \\
& +\mathbf{e}_{\varphi}\left[\frac{1}{R} \partial_{\varphi}-\frac{1}{\mathcal{F}^{2}}\left(\frac{1}{R} \partial_{\varphi}+\frac{1}{\bar{q} R_{0}} \partial_{\theta}\right)\right]
\end{aligned}
$$

where $\mathbf{b}$ is the unit vector in the equilibrium magnetic field direction, $\mathbf{e}_{r}$ is the unit vector in the radial direction and $\mathcal{F}=\sqrt{1+\epsilon^{2} / \bar{q}^{2}}$. We split the distribution function in a perturbed distribution $(f)$ and a Maxwellian background $\left(F_{M}\right)$ with no parallel flow

$$
F_{M}=\frac{n_{0}}{(2 \pi T / m)^{3 / 2}} \exp \left[-\frac{m v_{\|}^{2}+2 \mu B}{2 T}\right]
$$

where $n_{0}$ and $T$ are the ion equilibrium density and temperature respectively. We assume $f$ to be of order $\rho_{*}$ compare to $F_{M}$, i.e. $f \approx \rho_{*} F_{M}$ with $\rho_{*}=\rho / R_{0} \ll 1$ where $\rho=v_{\perp} / \omega_{c}$ is the ion gyroradius, $\omega_{c}=Z e B / m$ the ion cyclotron frequency, $e$ is the elementary electric charge and $Z$ is the ion charge number. We consider the perturbed electrostatic potential $\phi$ to be of the same order as $f$. The linearised gyro-kinetic equation for $f$, in the $\delta f$-approximation, assuming no background electrostatic potential, is then given by (see e.g. [21])

$$
\begin{aligned}
\frac{\partial f}{\partial t} & +\left(\mathbf{v}_{\|}+\mathbf{v}_{g k}\right) \cdot \nabla f-\frac{\mu}{m} \mathbf{b} \cdot \nabla B \frac{\partial f}{\partial v_{\|}}= \\
& -\mathbf{v}_{E} \cdot\left[\frac{\nabla n_{0}}{n_{0}}+\left(\frac{m v^{2}}{2 T}-\frac{3}{2}\right) \frac{\nabla T}{T}\right] F_{M}+ \\
& -\frac{Z e}{T} F_{M}\left(\mathbf{v}_{\|}+\mathbf{v}_{g k}\right) \cdot \nabla[G(\phi)]
\end{aligned}
$$

where $v^{2}=v_{\|}^{2}+v_{\perp}^{2}$ and $G(\cdot)$ indicates the gyroaverage operator. The parallel and drift velocities 
vectors are given by

$$
\begin{aligned}
\mathbf{v}_{\|} & =\mathbf{b} v_{\|} \\
\mathbf{v}_{E} & =\frac{\mathbf{b} \times \nabla[G(\phi)]}{B} \\
\mathbf{v}_{g k} & =\frac{m v_{\|}^{2}+\mu B}{Z e B} \frac{\mathbf{b} \times \nabla B}{B}
\end{aligned}
$$

We use the ballooning representation [22]. We find a representation for $f$ and $\phi$ following a calculation presented in $[23,24]$. We exploit the strong separation between parallel and perpendicular dynamics $\left(v_{\|} \approx v_{t h}\right.$ and $v_{D} \approx \rho_{*} v_{t h}$ where $v_{D}$ indicates the modulus of the drift velocity and $v_{t h}=\sqrt{2 T / m}$ is the ion thermal velocity) to construct an eikonal solution as a perturbation series with ordering parameter $\rho_{*}$ that determines the rapidly varying perpendicular mode structure. To lowest order in $\rho_{*}$, we look for stationary solutions $f^{(0)}$ with no spatial variation along the equilibrium magnetic field, therefore we have

$$
\mathbf{v}_{\|} \cdot \nabla f^{(0)}=0
$$

The system is periodic in the toroidal direction, then using Eq. (3), we can integrate Eq. (7) between $\theta_{0}$ and $\theta$ to obtain

$$
f^{(0)}=e^{i n\left[\varphi-\bar{q} g\left(r, \theta, \theta_{0}\right)\right]}
$$

where $n$ is the toroidal mode number and

$$
g\left(r, \theta, \theta_{0}\right)=\int_{\theta_{0}}^{\theta} \frac{d \bar{\theta}}{1+\epsilon \cos \bar{\theta}}
$$

where $\bar{\theta}$ is the integration variable and the indefinite integral is given by [25]

$$
\begin{aligned}
& \int \frac{d \bar{\theta}}{1+\epsilon \cos \bar{\theta}}= \\
& =\frac{2}{\sqrt{1-\epsilon^{2}}} \arctan \left[\sqrt{\frac{1-\epsilon}{1+\epsilon}} \tan \left(\frac{\bar{\theta}}{2}\right)\right]
\end{aligned}
$$

The first order solution in $\rho_{*}$ is assumed to be of the form $f^{(1)}=\hat{F}\left(t, \mathbf{X}, v_{\|}, \mu\right) f^{(0)}$, where $\hat{F}$ is a slowly varying (i.e. $\nabla_{\perp} \hat{F} \ll \nabla_{\perp} f^{(0)}$ ) envelope function. In this paper, the discussion is focused on the problematic of treating the system analytically. In this regard, we consider the simplified case where the only resonance considered is the one due to the magnetic field inhomogeneities. This means that we do not consider the effect of parallel dynamics, i.e. we neglect in Eq. (5) the $\mathbf{v}_{\|} \cdot \nabla$ terms. Therefore, assuming the time dependence to be such that
$\hat{F}\left(t, \mathbf{X}, v_{\|}, \mu\right)=\hat{f}\left(\mathbf{X}, v_{\|}, \mu\right) e^{-i \omega t}$, we find that $f$ can be written as

$$
f=\hat{f}\left(\mathbf{X}, v_{\|}, \mu\right) e^{-i \omega t+i n\left[\varphi-\bar{q} g\left(r, \theta, \theta_{0}\right)\right]}
$$

and similarly for the electrostatic potential

$$
\phi=\hat{\phi}(\mathbf{X}) e^{-i \omega t+i n\left[\varphi-\bar{q} g\left(r, \theta, \theta_{0}\right)\right]}
$$

where $\omega=\omega_{r}+i \gamma$ with $\omega_{r}$ and $\gamma$ respectively frequency and growth rate. Inserting these expressions in Eq. (5) and using Eq. (3) we obtain

$$
\begin{aligned}
& \left(\omega-\omega_{g k}\right) f+\frac{\mu}{m} \mathbf{b} \cdot \nabla B \frac{\partial f}{\partial v_{\|}}= \\
& =-\frac{Z e}{T} F_{M}\left(\omega_{*}-\omega_{g k}\right) J_{0}\left(k_{\perp} \rho\right) \phi
\end{aligned}
$$

where the diamagnetic drift frequency $\omega_{*}$, the drift frequency due to the magnetic field inhomogeneities $\omega_{g k}$ and the magnitude of the perpendicular (to the magnetic field) component of the wave vector $k_{\perp}$ are given by

$$
\begin{aligned}
\omega_{*}= & -\frac{T}{Z e B^{2}} \frac{B_{0} R_{0}}{R} \frac{n \bar{q}}{r}\left(\partial_{\theta} g+\frac{r \epsilon}{\bar{q}^{2} R}\right) . \\
& \cdot\left[\frac{\nabla n_{0}}{n_{0}}+\left(\frac{m v^{2}}{2 T}-\frac{3}{2}\right) \frac{\nabla T}{T}\right] \\
\omega_{g k}= & \frac{m v_{\|}^{2}+\mu B}{Z e B^{3}} \frac{B_{0} R_{0}}{R} \frac{n \bar{q}}{r} . \\
& \cdot\left[\partial_{\theta} B \frac{\partial_{r}(\bar{q} g)}{\bar{q}}-\partial_{r} B\left(\partial_{\theta} g+\frac{r \epsilon}{\bar{q}^{2} R}\right)\right] \\
k_{\perp}= & \frac{n \bar{q}}{r} \sqrt{\left(\frac{r \partial_{r}(\bar{q} g)}{\bar{q}}\right)^{2}+\left(\partial_{\theta} g\right)^{2}+\left(\frac{r}{\bar{q} R}\right)^{2}}
\end{aligned}
$$

The gyro-average operation has been approximated as $G(\phi)=J_{0}\left(k_{\perp} \rho\right) \phi$ where $J_{0}$ is the zeroth order Bessel function of the first kind This approximation is valid since in the local limit the turbulence is considered homogeneous in the perpendicular (to the magnetic field) directions, therefore periodic boundary conditions can be employed and a Fourier decomposition can be used (see e.g. [23, 26]). The approximation $\nabla_{\perp}\left[J_{0}\left(k_{\perp} \rho\right) \phi\right]=J_{0}\left(k_{\perp} \rho\right) \nabla_{\perp} \phi$ has also been employed. We choose to locally evaluate Eq. (13) at $\theta=\theta_{0}=0$ to obtain

$$
f=-\frac{Z e}{T} F_{M} \frac{\omega_{*}-\omega_{g k}}{\omega-\omega_{g k}} J_{0}\left(k_{\theta} \rho\right) \phi
$$

where the last term of the left hand side of Eq. (13) does not appear since at $\theta=0$ we have $\mathbf{b} \cdot \nabla B=0$, and we have defined $k_{\theta}=k_{\perp}\left(\theta=\theta_{0}=0\right)$. The drift frequencies and the perpendicular component 
of the wave vector take the form

$$
\begin{aligned}
\omega_{*} & =k_{\theta} \rho_{t h} \frac{v_{t h}}{R_{0}}\left[\frac{R}{L_{n}}+\left(\frac{m v^{2}}{2 T}-\frac{3}{2}\right) \frac{R}{L_{T}}\right] \mathcal{L}_{*} \\
\omega_{g k} & =k_{\theta} \rho_{t h} \frac{v_{t h}}{R_{0}} \frac{m v_{\|}^{2}+\mu B}{2 T} \mathcal{L}_{g k} \\
k_{\theta} & =\frac{n \bar{q}}{r} \frac{\mathcal{F}}{1+\epsilon}
\end{aligned}
$$

where $\rho_{t h}=v_{t h} / \omega_{c 0}$ is the thermal ion gyro-radius with $\omega_{c 0}=Z e B_{0} / m$ is the ion cyclotron frequency at the magnetic axis. The normalised inverse background density and temperature gradient lengths are respectively defined as $R / L_{n}=-R_{0} \nabla n_{0} / n_{0}$ and $R / L_{T}=-R_{0} \nabla T / T$. The geometry coefficients $\mathcal{L}_{*}$ and $\mathcal{L}_{g k}$ are given by

$$
\begin{aligned}
\mathcal{L}_{*} & =\frac{1+\epsilon}{2 \mathcal{F}} \\
\mathcal{L}_{g k} & =\frac{1}{\mathcal{F}}-\frac{\epsilon}{q^{2} \mathcal{F}^{3}(1-\epsilon)}\left(1-\hat{s}+\frac{\epsilon^{2}}{1-\epsilon^{2}}\right)
\end{aligned}
$$

where $\hat{s}=(r / q) d q / d r$ is the magnetic shear. The dispersion relation is obtained coupling Eq. (15) to the quasi-neutrality equation. The quasi-neutrality equation for one single kinetic ion species and adiabatic electrons can be written in the form

$$
\begin{aligned}
& \frac{2 \pi B}{m} \int d v_{\|} d \mu\left[Z J_{0}\left(k_{\theta} \rho\right) f+\right. \\
& \left.\quad+\frac{Z^{2} e}{T} F_{M}\left(J_{0}^{2}\left(k_{\theta} \rho\right)-1\right) \phi\right]=\frac{e}{T_{e}} n_{0 e} \phi
\end{aligned}
$$

see [23] for its derivation. The factor $2 \pi B / m$, appearing in front of the velocity space integral, is due to the change of variables $\int d^{3} v=$ $2 \pi \int d v_{\|} v_{\perp} d v_{\perp}=2 \pi B / m \int d v_{\|} d \mu$. The last two terms of the left hand side account for polarisation effect, the right hand side is the electrons adiabatic response with $n_{0 e}$ and $T_{e}$ the electron equilibrium density and temperature respectively. Inserting Eq. (15) into Eq. (18), assuming $n_{0 e}=n_{0}$, we finally obtain the dispersion relation

$$
\frac{2 \pi B}{m} \int d v_{\|} d \mu D\left(v_{\|}, \mu, \omega\right)+S=0
$$

The first term of Eq. (19) is the velocity space integral of the resonant part $D\left(v_{\|}, \mu, \omega\right)$ given by

$$
D\left(v_{\|}, \mu, \omega\right)=F_{M} \frac{\omega_{*}-\omega_{g k}}{\omega-\omega_{g k}} J_{0}^{2}\left(k_{\theta} \rho\right)
$$

The second term of Eq. (19) is given by

$$
S=n_{0}\left(1+\frac{\tau}{Z^{2}}\right)-\Gamma_{0}
$$

where $\tau$ is the ion to the electron temperature ratio and $\Gamma_{0}=2 \pi B / m \int d v_{\|} d \mu\left[F_{M} J_{0}^{2}\left(k_{\theta} \rho\right)\right]=$ $n_{0} I_{0}\left(2 \delta^{2}\right) e^{-2 \delta^{2}}$, with $I_{0}$ the zeroth order modified Bessel function of the first kind, having defined $\delta=k_{\theta} \rho_{t h} /(2 b)$ where $b=B / B_{0}$.

The dispersion relation Eq. (19) is an integral equation to be solved for $\omega$ (note that it does not provide a solution for the envelope $\hat{\phi}$ since the parallel dynamics effect is neglected). The difficulty is obviously the evaluation of the velocity space integral of $D\left(v_{\|}, \mu, \omega\right)$. There are different methods that can be used to solve the problem. The HFA tackles the problem assuming $\omega_{g k}<|\omega|$, providing the convergence of the series $\sum_{n}\left(\omega_{g k} / \omega\right)^{n}$ such that one can write

$$
\frac{\omega_{*}-\omega_{g k}}{\omega-\omega_{g k}}=\frac{\omega_{*}-\omega_{g k}}{\omega} \lim _{n \rightarrow \infty} \sum_{l=0}^{n}\left(\frac{\omega_{g k}}{\omega}\right)^{l}
$$

then the integrals in velocity space can be performed analytically and the dispersion relation, once the sum is truncated to a finite $n$, becomes a polynomial equation of order $n+1$ in $\omega$ under the assumption $\omega \neq 0$. However, Eq. (19) can also be solved numerically for a chosen set of plasma parameters, without any series expansion: the numerical integration in velocity space is performed scanning over a range of values for $\omega_{r}$ and $\gamma$ (imposing $\gamma \neq 0$ to avoid the singularity). Then, interpolating the results of the integrations in the $\left(\omega_{r}, \gamma\right)$ plane (we used here an interpolation method that works by fitting cubic polynomial curves between successive data points), the solutions of the dispersion relation are found as shown in $[17,18]$ for the case of the EGAM, i.e. plotting the inverse of the modulus of the left hand side of Eq. (19) in the $\left(\omega_{r}, \gamma\right)$ plane and looking for its poles. In the following, we will refer to this procedure as the Interpolation Method (IM). The solution to the problem can be also obtained running a gyro-kinetic code. Furthermore, the physics of the ITG mode can be investigated using a low field side gyro-fluid model. We build the gyro-fluid model following the procedure described in [21], introducing Finite Larmor Radius (FLR) and polarization effects. The low field side gyro-fluid equations for perturbed density and temperature are obtained from Eq. (13) evaluated at $\theta=\theta_{0}=0$ by taking the appropriate moments, the system is then closed by Eq. (18). Defining the average

$$
\{G\}_{F}=\int d^{3} v G F
$$

the integrals over the Maxwellian needed for the 
derivation are given by

$$
\begin{aligned}
\left\{J_{0}\left(k_{\theta} \rho\right)\right\}_{F_{M}} & =n_{0} e^{-\delta^{2}} \\
\left\{m v_{\perp}^{2} J_{0}\left(k_{\theta} \rho\right)\right\}_{F_{M}} & =2 n_{0} T\left(1-\delta^{2}\right) e^{-\delta^{2}} \\
\left\{m v_{\|}^{2} J_{0}\left(k_{\theta} \rho\right)\right\}_{F_{M}} & =n_{0} T e^{-\delta^{2}} \\
\left\{m^{2} v_{\perp}^{4} J_{0}\left(k_{\theta} \rho\right)\right\}_{F_{M}} & =8 n_{0} T^{2}\left(1-2 \delta^{2}+2 \delta^{4}\right) e^{-\delta^{2}} \\
\left\{m^{2} v_{\|}^{4} J_{0}\left(k_{\theta} \rho\right)\right\}_{F_{M}} & =3 n_{0} T^{2} e^{-\delta^{2}} \\
\left\{m^{2} v_{\|}^{2} v_{\perp}^{2} J_{0}\left(k_{\theta} \rho\right)\right\}_{F_{M}} & =2 n_{0} T^{2}\left(1-\delta^{2}\right) e^{-\delta^{2}} \\
\left\{J_{0}^{2}\left(k_{\theta} \rho\right)\right\}_{F_{M}} & =n_{0} I_{0}\left(2 \delta^{2}\right) e^{-2 \delta^{2}}
\end{aligned}
$$

The moments of the perturbed distribution are given in Eq. (80) of [21], with the addition of

$$
\left\{J_{0}\left(k_{\theta} \rho\right)\right\}_{f}=n_{0} \tilde{n} e^{-\delta^{2}}
$$

where $\tilde{n}$ is the perturbed density normalised to $n_{0}$. Using the relations above the gyro-fluid equations can be written in the form

$$
\begin{aligned}
& \omega_{N} \tilde{n}-k \mathcal{L}_{g k}(\tilde{n}+\tilde{T})=k e^{-\delta^{2}} A \phi_{N} \\
& 3 \omega_{N} \tilde{T}-k \mathcal{L}_{g k}(2 \tilde{n}+7 \tilde{T})=k e^{-\delta^{2}} B \phi_{N}
\end{aligned}
$$

where $\tilde{T}$ is the perturbed temperature normalised to the background temperature, $\omega_{N}=\left(R_{0} / v_{t h}\right) \omega$, $k=k_{\theta} \rho_{t h}, \phi_{N}=(Z e / T) \phi$, and we have defined the quantities

$$
\begin{aligned}
A= & \mathcal{L}_{g k}\left(1-\frac{\delta^{2}}{2}\right)-\mathcal{L}_{*}\left(\frac{R}{L_{n}}-\delta^{2} \frac{R}{L_{T}}\right) \\
B= & \mathcal{L}_{g k}\left(2-4 \delta^{2}+\delta^{4}\right)+ \\
& -\mathcal{L}_{*}\left[\frac{R}{L_{T}}\left(3-4 \delta^{2}+2 \delta^{4}\right)-2 \delta^{2} \frac{R}{L_{n}}\right]
\end{aligned}
$$

The quasi-neutrality equation is given by

$$
n_{0} \tilde{n} e^{-\delta^{2}}=S \phi_{N}
$$

where $S$ is given in Eq. (21). Solving the system of Eq. (26)-(28) one obtains the dispersion relation as a second order polynomial equation for $\omega_{N}$

$$
\begin{aligned}
\omega_{N}^{2} & -\omega_{N} k\left(\frac{10}{3} \mathcal{L}_{g k}+A \frac{n_{0}}{S} e^{-2 \delta^{2}}\right)+ \\
& +\frac{1}{3} k^{2} \mathcal{L}_{g k}\left[5 \mathcal{L}_{g k}+\frac{n_{0}}{S} e^{-2 \delta^{2}}(7 A-B)\right]=0
\end{aligned}
$$

In the rest of the paper, all quantities are presented in normalised units. The velocity space coordinates are normalised to the ion thermal velocity such that $v_{\|}=v_{\| N} v_{t h}$ and $\mu=\mu_{N} m v_{t h}^{2} / B_{0}$. Lengths are

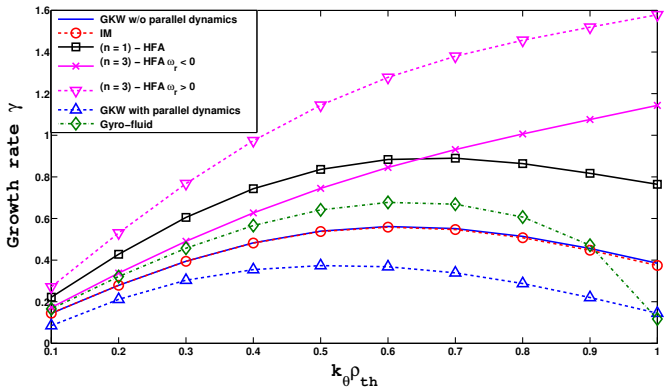

(a) Growth rate $\gamma$

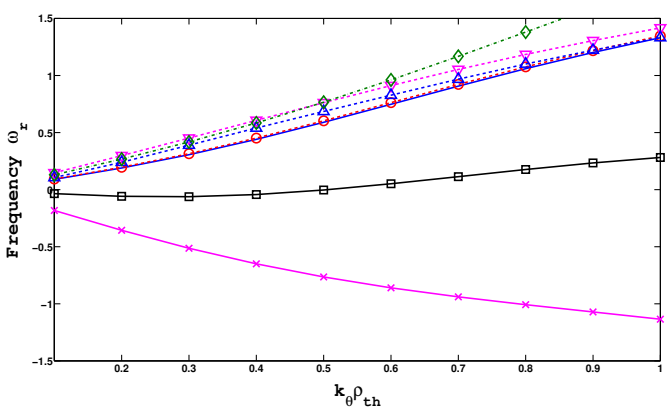

(b) Frequency $\omega_{r}$

Figure 1: Growth rate $\gamma$ and frequency $\omega_{r}$ as a function of $k_{\theta} \rho_{t h}$ for the Waltz standard case. Bluesolid: GKW using one point in the field line direction (i.e. suppressing the parallel dynamics). Blue-dashed-'triangles': GKW using more than one point in the field line direction (including the parallel dynamics). Red-dashed-'circles': using the IM. Black-solid-'squares': HFA cut to 1 st order $(n=1$ in Eq. (22)). Pink-solid-'x': HFA cut to 3rd order $\left(n=3\right.$ in Eq. (22)) with $\omega_{r}<0$. Pink-solid'inverted-triangles': HFA cut to 3rd order $(n=3$ in Eq. (22)) with $\omega_{r}>0$. Green-dashed-dotted'diamonds': gyro-fluid model in Eq. (29).

normalised to the tokamak major radius $R_{0}$. Frequencies are then normalised to $v_{t h} / R_{0}$. For simplicity in the notation the subscript $N$ will be suppressed in the following.

Fig. 1 shows a comparison of the different methods mentioned above to calculate the growth rate $(\gamma)$ and frequency $\left(\omega_{r}\right)$ as a function of $k_{\theta} \rho_{t h}$ for the Waltz standard case [27]: ion temperature gradient length $R / L_{T}=9.0$, density gradient length $R / L_{n}=3.0$, electron and ion temperature $T_{e}=T_{i}$ $(\tau=1)$, safety factor $q=2.0$, magnetic shear $\hat{s}=1.0$, ion charge number $Z=1$ and inverse aspect ratio $\epsilon=0.16$. The blue-solid line is obtained running the gyro-kinetic code GKW [20] using one point only in the field line direction (i.e. suppressing parallel dynamics effects), the blue- 
dashed-'triangles' line running GKW using more than one point in the field line direction (including parallel dynamics effects), the red-dashed-'circles' using the IM, the black-solid-'squares' employing the HFA cut to 1 st order $(n=1$ in Eq. (22)), the pink-solid-' $x$ ' employing the HFA cut to 3rd order $\left(n=3\right.$ in Eq. (22)) with $\omega_{r}<0$, the pink-solid'inverted-triangles' employing the HFA cut to 3rd order $\left(n=3\right.$ in Eq. (22)) with $\omega_{r}>0$ and the green-dashed-dotted-'diamonds' solving the gyrofluid model in Eq. (29).

The IM gives the same result as the gyro-kinetic code GKW without parallel dynamics, showing that the IM applied to Eq. (19) can be used as a verification tool for gyro-kinetic codes as well as for the investigation of fundamental physical mechanisms. It can be observed that including parallel dynamics in GKW, i.e. introducing more than one point in the parallel direction, tends to stabilize the mode over the whole spectrum, having a stronger impact at higher $k_{\theta} \rho_{t h}$, without considerably affecting the frequency. The gyro-fluid model captures the qualitative behaviour of the system, but overestimates the growth rate for $0.1 \leq k_{\theta} \rho_{t h} \leq 0.9$ and underestimates it at $k_{\theta} \rho_{t h}=1.0$, and it overestimates the frequency for each value of $k_{\theta} \rho_{t h}$. In the case of the HFA, the number of solutions is equal to the order of the polynomial equation obtained from the expansion and in Fig. 1 only the unstable solutions $(\gamma>0)$ are shown: the order of the polynomial is $n+1$, so we have one unstable solution for $n=1$ and two unstable solutions for $n=3$ (note that this is true if $\gamma \neq 0$ ). It is clear that the HFA captures the qualitative behaviour of the system only in some cases, and it is in obvious disagreement with the other methods: it largely overestimates the growth rate and gives solutions with negative values for the frequency (meaning that the mode should drift in the electron diamagnetic drift direction) which are not found by the other methods. It is then not clear which solution should be chosen as the physical one. More importantly, it has to be noticed that increasing the order of the expansion of the HFA does not improve the result: for this set of parameters it is not possible to get convergence to the other methods results, which however one would expect to be the case when applying this kind of approximation.

To understand the behaviour of the solution given by the HFA, one has to look into the condition under which the approximation is applied. The HFA assumes $\omega_{g k}<|\omega|$, this relation selects

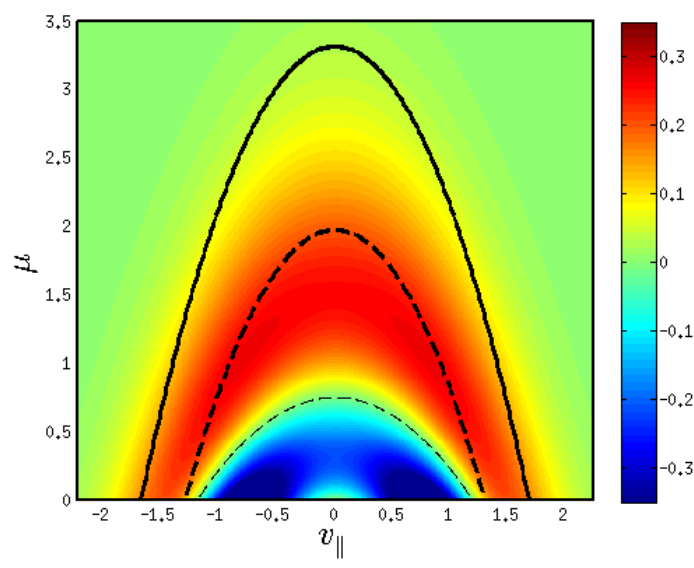

(a) Waltz standard case $\left(R / L_{T}=9\right)$

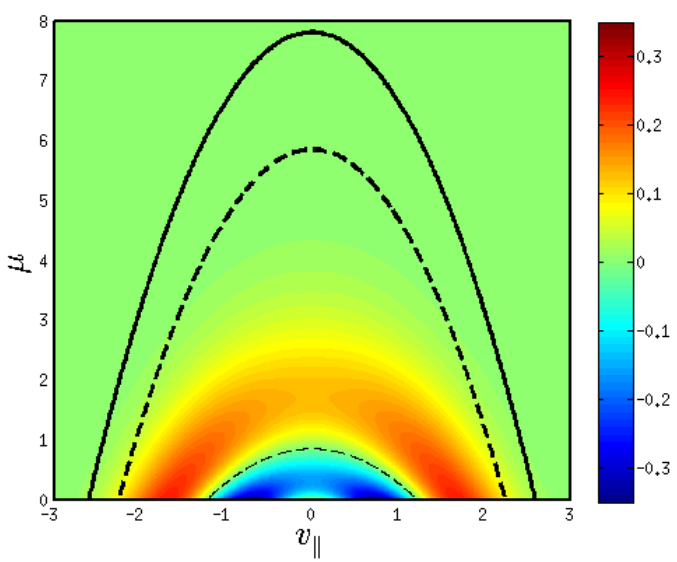

(b) Waltz standard case with $R / L_{T}=50$

Figure 2: Ion contribution to the growth rate at $k_{\theta} \rho_{t h}=0.15$ in velocity space for $R / L_{T}=9$ and $R / L_{T}=50$. Black-thin-dashed: zeros of the function $\left(\omega_{*}=\omega_{g k}\right)$, it is the boundary between the stabilizing (negative values) and destabilizing (positive values) region of the velocity space. Blackthick-dashed: boundary given by Eq (30), it is the limit of applicability of the HFA. Black-thick-solid: boundary given by Eq (33), it is the limit of applicability of the IHFA.

the velocity space region given by

$$
\mu<\frac{1}{b}\left(-v_{\|}^{2}+\frac{|\omega|}{\mathcal{L}_{g k}}\right)
$$

The HFA expansion given in Eq. (22) can be strictly applied only over this region. Outside of it the series on the right hand side of Eq. (22) does not converge to the function on the left hand side.

We investigate the physical meaning of the condition given in Eq. (30) performing a velocity space analysis of the wave-particle energy exchange. The 
contributions of particles to the linear growth rate in velocity space can be identified linking the mode growth rate to the work done by the perturbed electric field on the particles starting from the energy conservation property of the Vlasov-Poisson system of equations [28]. In our case we find

$$
\gamma=\frac{1}{S} \int d v_{\|} d \mu \omega_{g k} \Re\left[i D\left(v_{\|}, \mu, \omega\right)\right]
$$

Notice that this equation can not be used by itself to calculate the growth rate $\gamma$, since the unknown $\omega_{r}$ also appears on the right hand side. Its consistency can be easily shown using Eq. (19) which states that $\int d v_{\|} d \mu \Im\left[D\left(v_{\|}, \mu, \omega\right)\right]=0$, then the real part of Eq. (19) is exactly Eq. (31). We use Eq. (31) for diagnostic purposes once $\omega_{r}$ and $\gamma$ have been already obtained.

Fig. 2 shows the ion contribution to the growth rate at $k_{\theta} \rho_{t h}=0.15$ in velocity space, strictly speaking it shows the integrand of Eq. (31) divided by $S$. We chose the value $k_{\theta} \rho_{t h}=0.15$ since in nonlinear simulations this is often the mode with the highest intensity. The-black-thin-dashed line indicates the zeros of the function plotted $\left(\omega_{*}=\omega_{g k}\right)$, it is the boundary between the stabilizing (negative values) and destabilizing (positive values) region of the velocity space, the black-thick-dashed line is the boundary given by Eq (30), it is the limit of applicability of the HFA, the black-thick-solid line is the boundary given by Eq (33), it is the limit of applicability of the IHFA discussed later.

For the Waltz standard case in Fig. 2a, we notice that the HFA is valid in a region which is much smaller than the area where particles contribute to the instability. This explains why integrating over the whole velocity space when applying the HFA gives results that do not converge to the other methods: outside the region embedded in the black thick dashed line the series in Eq. (22) does not converge to the function on the left hand side. This is a problem related to the range of parameters considered, since the HFA validity region does not include a large portion of the velocity space where the particles have a considerable contribution to the growth rate. It is well known that the HFA works well in the case of very high temperature gradients, the reason being that the area where the particles contribute to the instability remains almost the same as for the case of small temperature gradients, i.e. $-2.5<v_{\|}<2.5$ and $0<\mu<3.5$, but the HFA validity region increses enough to contain it. Fig. $2 \mathrm{~b}$ shows that this is the case for $k_{\theta} \rho_{t h}=0.15$ and $R / L_{T}=50$ as choice of parameters (higher $k_{\theta} \rho_{t h}$ need higher $\left.R / L_{T}\right)$.
It is possible to improve the HFA noticing that

$$
\frac{\omega_{*}-\omega_{g k}}{\omega-\omega_{g k}}=\frac{\left(\omega_{*}-\omega_{g k}\right)\left(\omega_{r}-\omega_{g k}-i \gamma\right)}{\left(\omega_{r}^{2}+\gamma^{2}\right)\left(1-\frac{2 \omega_{g k} \omega_{r}-\omega_{g k}^{2}}{\omega_{r}^{2}+\gamma^{2}}\right)}
$$

then, provided that $\left|2 \omega_{g k} \omega_{r}-\omega_{g k}^{2}\right|<\omega_{r}^{2}+\gamma^{2}$, i.e.

$$
\mu<\frac{1}{b}\left(-v_{\|}^{2}+\frac{\omega_{r}+\sqrt{2 \omega_{r}^{2}+\gamma^{2}}}{\mathcal{L}_{g k}}\right)
$$

we can write

$$
\frac{1}{1-\frac{2 \omega_{g k} \omega_{r}-\omega_{g k}^{2}}{\omega_{r}^{2}+\gamma^{2}}}=\lim _{n \rightarrow \infty} \sum_{l=0}^{n}\left(\frac{2 \omega_{g k} \omega_{r}-\omega_{g k}^{2}}{\omega_{r}^{2}+\gamma^{2}}\right)^{l}
$$

which allows to analytically perform the velocity space integral of $D\left(v_{\|}, \mu, \omega\right)$. As shown in Fig. 2, the region of validity of this expansion, given by the black thick solid line, is substantially wider than the one of the HFA. We therefore refer to the expansion given in Eq. (34) as IHFA (Improved High Frequency Approximation). In particular, one has to notice that the region of validity almost includes the whole region where the particles contribute to the instability even for the Waltz standard case. However, it is important to stress that one needs to be very careful when using either the HFA or the IHFA when integrating over the whole velocity space (i.e. including regions where the approximations are not valid) for any choice of plasma parameters, even in the case of very high temperature gradients: outside the region of validity, the expansion can always be found to diverge from the function it approximates provided the order is high enough (for example, it can be checked that for $R / L_{T}=50$ one needs $n \leq 2$, the upper limit for $n$ increases with increasing $\left.R / L_{T}\right)$. Furthermore, we point out that while $D\left(v_{\|}, \mu, \omega\right)$ is an analytic function of $\omega$ over the whole velocity space (i.e. it satisfies the Cauchy-Riemann equations $\partial_{\omega_{r}} \Re[D]=\partial_{\gamma} \Im[D]$ and $\partial_{\omega_{r}} \Im[D]=-\partial_{\gamma} \Re[D]$ for each $v_{\|}$and $\mu$ ), the IHFA truncated at any order $n$ is not. This is not relevant for the results presented in this paper, but it has to be taken into account in applications where the analyticity is a necessary condition. For example, analyticity is sometimes used to find the zeros of the dispersion relation employing techniques based on generalized Nyquist method [29].

In the case of tokamak relevant parameters (the Waltz standard case), both the HFA and the IHFA can be strictly applied only over their respective region of validity for any order $n$ of the expansion, since outside the region of validity they immediately diverge from the functions to be approximated. The order needed to have a satisfactory 


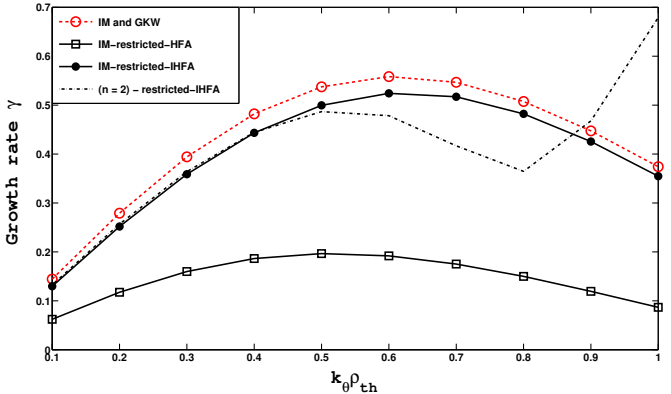

(a) Growth rate $\gamma$

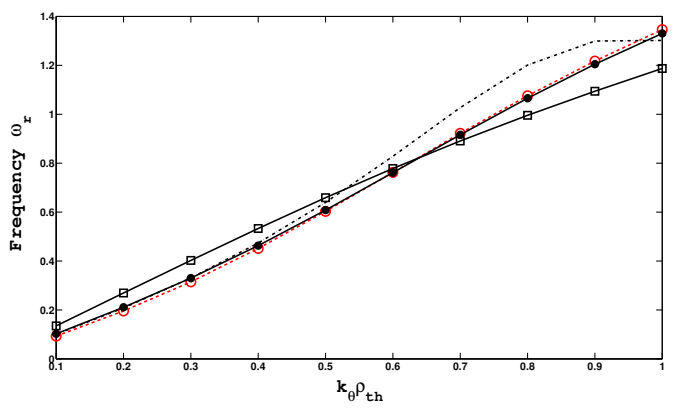

(b) Frequency $\omega_{r}$

Figure 3: Growth rate $\gamma$ and frequency $\omega_{r}$ as a function of $k_{\theta} \rho_{t h}$ for the Waltz standard case. Reddashed-'circles': IM integrating over the whole velocity space. Black-solid-'squares': IM integrating over the reduced HFA velocity space given by Eq. (30). Black-solid-'full-circles': IM integrating over the reduced IHFA velocity space given by Eq. (33). Black-dash-dotted: using Eq. (34) with $n=2$ to analytically integrate $D\left(v_{\|}, \mu, \omega\right)$ over the reduced IHFA velocity space given by Eq. (33).

approximation within the validity region mainly depends on the values of $k_{\theta} \rho_{t h}$ and $R / L_{T}$ : fixing $k_{\theta} \rho_{t h}$ one needs higher $n$ at lower $R / L_{T}$, fixing $R / L_{T}$ one needs higher $n$ at higher $k_{\theta} \rho_{t h}$. The best result that one can potentially obtain properly applying the HFA and IHFA is considering the expansions with $n \rightarrow+\infty$ and integrating over the respective region of validity only. Fig. 3 shows the outcome of this procedure. The red-dashed-'circles' line is obtained using the IM integrating over the whole velocity space (shown for comparison). We solve the dispersion relation using the IM, without expanding $D\left(v_{\|}, \mu, \omega\right)$ (which is equivalent to considering $n \rightarrow+\infty)$ and restricting the integration domain as in Eq. (30) for the HFA (black-solid'squares') and as in Eq. (33) for the IHFA (blacksolid-'full-circles'). We will refer to these methods as IM-restricted-HFA and IM-restricted-IHFA respectively. The results are compared to the case where we use Eq. (34) with $n=2$ to analytically integrate $D\left(v_{\|}, \mu, \omega\right)$ over the reduced IHFA velocity space (black-dash-dotted). We will refer to this method as $(n=2)$-restricted-IHFA. Performing the same exercise for the HFA (not shown in the figure), i.e. using Eq. (22) with $n=2$, only stable modes $(\gamma=0)$ are found, in fact a much higher $n$ is needed in order to get a satisfactory approximation. Note that in these last two cases, in order to perform the integrals analytically, an approximation for the Bessel function needs to be chosen. Here we use $J_{0}^{2}\left(k_{\theta} \rho\right) \approx 1-\left(k_{\theta} \rho\right)^{2} / 2+3\left(k_{\theta} \rho\right)^{4} / 32$. This is a good approximation only for $k_{\theta} \rho<1.5$, which gives the condition $k_{\theta} \rho_{t h}<0.5$ since the maximum value of $\mu$ to be considered is $\mu_{\max }=3.5$ and $k_{\theta} \rho=k_{\theta} \rho_{t h} \sqrt{2 \mu / b}$. The effect of this condition will be clarified in the following. Integrating over the reduced velocity space, we find an analytical expression for the left hand side of Eq. (19) which generally does not have simple analytical solutions. The solutions need to be found as shown in $[17,18]$ for the case of the EGAM, i.e. plotting the inverse of the modulus of the left hand side of Eq. (19) in the $\left(\omega_{r}, \gamma\right)$ plane and looking for its poles (note that, compared to the IM, the advantage is that one does not need to perform the numerical integrations and the interpolation procedure). We provide below the two type of indefinite integrals needed to use the HFA and the IHFA over restricted regions of the velocity space. The integrals over $\mu$ are always of the form [25]

$$
\int d x x^{n} e^{-x}=-e^{-x} \sum_{k=0}^{n} \frac{n ! x^{n-k}}{(n-k) !}
$$

The integrals over $v_{\|}$are always of the form [30]

$$
\begin{aligned}
\int d x & x^{2 n} e^{-x^{2} / 2}= \\
= & \sqrt{2 \pi} \frac{(2 n-1) !}{(n-1) ! 2^{n}}\left[1+\operatorname{erf}\left(\frac{x}{\sqrt{2}}\right)\right]+ \\
& -e^{-x^{2} / 2} \sum_{k=0}^{n-1} \frac{(2 n-1) ! k ! 2^{k} x^{2 k+1}}{(2 k+1) !(n-1) ! 2^{n-1}}
\end{aligned}
$$

where $\operatorname{erf}(x)$ is the error function

$$
\operatorname{erf}(x)=\frac{2}{\sqrt{\pi}} \int_{0}^{x} d t e^{-t^{2}}
$$

For the Waltz standard case, the IM-restrictedHFA largely underestimate the growth rate and it is in good agreement with the other methods concerning the calculation of the frequency. We conclude that, if properly used, the HFA fails in 
quantitatively predicting the growth rate, but it shows the main physical features of the mode: a bell shape curve for $\gamma$ and a quasi-linear growth for $\omega_{r}$ as a function of $k_{\theta} \rho_{t h}$, however, it needs a high order approximation to treat the system analytically. The IM-restricted-IHFA is in satisfactory agreement with the IM and GKW, giving a small underestimation of $\gamma$ and exactly the same $\omega_{r}$. It can be used to analytically treat the system using reasonable order for the approximation $(n \leq 2$ and $J_{0}^{2}\left(k_{\theta} \rho\right)$ expanded to the 4 th order in $\left.k_{\theta} \rho\right)$ for wave lengths such that $k_{\theta} \rho_{t h} \leq 0.5$ the reason being related to both the choices of the value of $n$ and the approximation for the Bessel function: one can check that even in the case $n \rightarrow+\infty$ if $J_{0}^{2}\left(k_{\theta} \rho\right)$ is expanded to the 4 th order in $k_{\theta} \rho$ it is not possible to get agreement for $k_{\theta} \rho_{t h}>0.5$ with the IM. We stress the fact that for $k_{\theta} \rho_{t h}=0.15$ (highest intensity mode in non-linear simulations) the IHFA can be safely used.

To conclude our analysis, we calculate the temperature gradient threshold. Fig. 4 shows a scan over $R / L_{T}$ at fixed $k_{\theta} \rho_{t h}=0.15$ for the Waltz standard case comparing the different methods under analysis. The red-dashed-'circles' is obtained using the IM integrating over the whole velocity space, the black-solid-'full-circles' line using the IM integrating over the reduced IHFA velocity space given by Eq. (33), the black-dash-dotted line using Eq. (34) with $n=2$ to analytically integrate $D\left(v_{\|}, \mu, \omega\right)$ over the reduced IHFA velocity space given by Eq. (33), the green-dasheddotted-'diamonds' line solving the gyro-fluid model in Eq. (29) and the black-solid-'squares' line employing the HFA with $n=1$ integrating over the whole velocity space.

The value for the threshold given by the IM and $\mathrm{GKW}$ is $R / L_{T_{\text {crit }}} \approx 2.5$, the IM-restricted-IHFA slightly overestmates it, the error is bigger in the case of the $(n=2)$-IHFA (as mentioned above the accuracy of the analytical method at given $n$ drops at smaller $\left.R / L_{T}\right)$. Even though not applicable in this case, we show the result of the $(n=1)$-HFA for comparison, since this is the method usually employed for analytic calculations. It largely underestimates the threshold and gives negative values for the frequency over the whole scan. Furthermore, it can be shown that at higher $n$, the HFA finds unstable solution even for zero and negative $R / L_{T}$. Once again, we stress the fact that the application of the HFA should be restricted only to the range of parameters of its validity and still does not give quantitatively correct results. The gyro-fluid model gives the right qualitative behavior concerning the

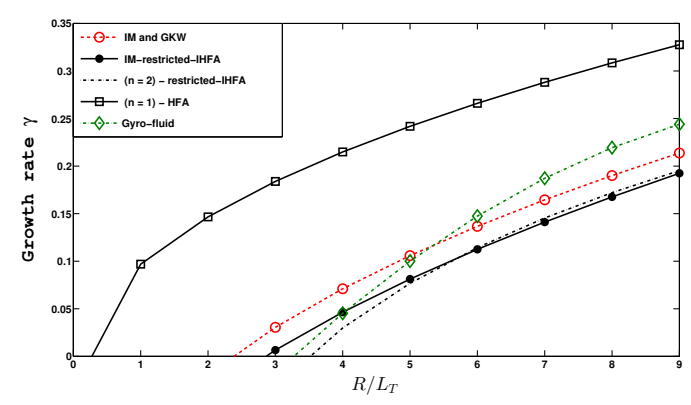

(a) Growth rate $\gamma$

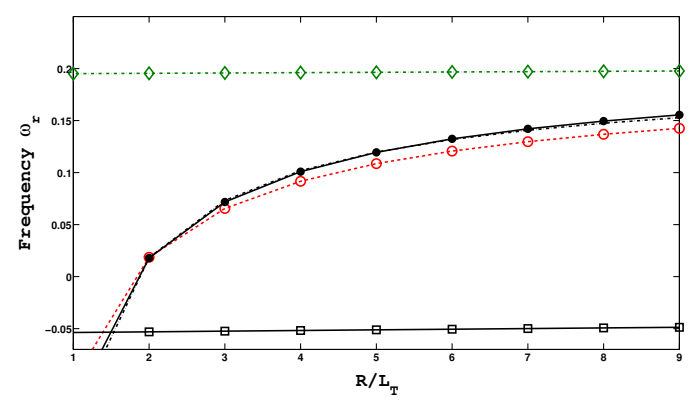

(b) Frequency $\omega_{r}$

Figure 4: Growth rate $\gamma$ and frequency $\omega_{r}$ as a function of $R / L_{T}$ at fixed $k_{\theta} \rho_{t h}=0.15$ for the Waltz standard case. Red-dashed-'circles': IM integrating over the whole velocity space. Black-solid-'fullcircles': IM integrating over the reduced IHFA velocity space given by Eq. (33). Black-dash-dotted: using Eq. (34) with $n=2$ to analytically integrate $D\left(v_{\|}, \mu, \omega\right)$ over the reduced IHFA velocity space given by Eq. (33). Black-solid-'squares': HFA with $n=1$ integrating over the whole velocity space. Green-dashed-dotted-'diamonds': gyro-fluid model in Eq. (29).

growth rate dependence on $R / L_{T}$, but it overestimates the threshold $\left(R / L_{T_{\text {crit }}} \approx 3.3\right)$ and it finds a constant value for the frequency.

We have presented an analysis of different methods that can be used to treat the electrostatic ITG instability in toroidal geometry without considering the effect of parallel dynamics. We have discussed the problematic of treating the system analytically. We have taken into consideration two already known methods: the HFA and the gyrofluid model, and we have proposed a new approximation: the IHFA. It is shown that one has to be very careful when applying any of these approximations. The main important role being played by the restriction imposed by the approximation over its validity region in velocity space. For tokamak relevant parameters, we find that the HFA can not be 
applied to obtain physically consistent results. The gyro-fluid model can be employed to analyse only the qualitative behaviour of the system. The IHFA can be safely used once the order for the expansions (both for $D\left(v_{\|}, \mu, \omega\right)$ and the Bessel function) are properly chosen.

\section{Acknowledgments}

This work has been carried out thanks to the support of the A*MIDEX project (ANR-11-IDEX0001-02) funded by the "Investissement d'Avenir" French Government Program, managed by the French National Research Agency (ANR). This work was granted acces to the HPC resources of Aix-Marseille Université financed by the project Equip@Meso (ANR-10-EQPX-29-01) of the program "Investissement d'Avenir" supervised by the Agence Nationale de la Recherche.

\section{References}

[1] F. Romanelli, Physics of Fluids B 1, 1018 (1989)

[2] F. Romanelli, Liu Chen, and S. Briguglio, Physics of Fluids B 3, 2496 (1991)

[3] C. Bourdelle, X. Garbet, F. Imbeaux, A. Casati, N. Dubuit and T. Parisot, Physics of Plasmas 14, 112501 (2007)

[4] A. Casati, Ph.D. dissertation, Université de Provence (Aix-Marseille I), 2009

[5] P. Cottier, C. Bourdelle, Y. Camenen, Ö.D. Gürcan, F.J. Casson, X. Garbet, P. Hennequin, and T. Tala, Angular momentum transport modeling: achievements of a gyrokinetic quasi-linear approach, PPCF, 56(1):015011 (2014)

[6] C. Bourdelle, Turbulent transport in tokamak plasmas: bridging theory and experiment. Habilitation é Diriger des Recherches, AixMarseille University (2015)

[7] J. Wesson, Tokamaks Second Edition, Oxford Science Publications, Clarendon Press-Oxford (1997)

[8] S. Brunner, Waves and Instabilities in Inhomogeneous Plasmas, Centre de Recherches en Physique de Plasmas, Association EuratomConfédération Suisse, Ecole Polytechnique Fédérale de Lausanne, CRPP-PPB, CH-1015
Lausanne, Switzerland https://crppwww. epfl.ch/ brunner/inhomoplasma.pdf

[9] G.W. Hammett, CMPD/CMSO Winter School UCLA 1/09/2007, PPPL http://w3.pppl.gov/ hammett/refs/ 2007/hammett_itginstability_lec.pdf

[10] R.G. Littlejohn, J. Plasma Phys. 29, 111 (1983)

[11] T.S. Hahm, Phys. Fluids 31, 043207 (1988)

[12] A. Brizard, Phys. Plasmas 41, 541 (1988)

[13] H. Sugama, Phys. Plasmas 7, 466 (2000)

[14] M.A. Beer, S.C. Cowley, and G.W. Hammett, Phys. Plasmas 2, 2687 (1995)

[15] X. Lapillonne, S. Brunner, T. Dannert, S. Jolliet, A. Marinoni, L. Villard, T. Goerler, F. Jenko, and F. Merz, Phys. Plasmas 16, 032308 (2009).

[16] D. Zarzoso, Y. Sarazin, X. Garbet, R. Dumont, A. Strugarek, J. Abiteboul, T. CartierMichaud, G. Dif-Pradalier, P. Ghendrih, V. Grandgirard, G. Latu, C. Passeron, and O. Thomine, Phys. Rev. Lett. 110, 125002 (2013).

[17] D. Zarzoso, A. Biancalani, A. Bottino, Ph. Lauber, E. Poli, J. B. Girardo, X. Garbet and R.J. Dumont, Nuclear Fusion 54, 103006 (2014)

[18] J.B. Girardo, D. Zarzoso, R. Dumont, X. Garbet, Y. Sarazin, and S. Sharapov, Physics of Plasmas (1994-present) 21, 092507 (2014)

[19] R.A. Treumann and W. Baumjohann, Advanced Space Plasma Physics, Imperial College Press

[20] A.G. Peeters, Y. Camenen, F.J. Casson, W.A. Hornsby, A.P. Snodin, D. Strintzi and G. Szepesi, Comp. Phys. Comm. 180, 2650 (2009)

[21] A.G. Peeters, D. Strintzi, Y. Camenen, C. Angioni, F.J. Casson, W.A. Hornsby and A.P. Snodin, Phys. Plasmas 16, 042310 (2009)

[22] J.W. Connor, R.J. Hastie, and J.B. Taylor, Phys. Rev. Lett. 40, 396 (1978)

[23] F.J. Casson, Ph.D. dissertation, University of Warwick, 2011 
[24] https://bitbucket.org/gkw/gkw/src/

a7c683414e3ba4384dadaa78ff32e769ded2f3c2/

doc/tex/Ballooning.tex?at=develop\&

fileviewer=file-view-default

[25] I.S. Gradshteyn and I.M. Ryzhik, Table of Integrals, Series, and Products 4th ed. Academic Press (1965)

[26] N. Crouseilles, M. Mehrenberger and H. Sellama, Commun. Comput. Phys., Vol. 8, No. 3, pp. 484-510 (2010)

[27] R.E. Waltz, G.D. Kerbel, and J. Milovich, Phys. Plasmas 1, 2229 (1994)

[28] R. Hatzky, T.M. Tran, A. Könies, R. Kleiber and S.J. Allfrey. Phys. Plasmas 9, 898 (2002)

[29] B. Davies, J. Comp. Phys., 663649 (1986)

[30] D. B. Owen, A table of normal integrals, Communications in Statistics - Simulation and Computation, 9:4, 389-419 (1980) 